\title{
High Resolution Fourier-Transform Microspectroscopy Based on Spiral Silicon Waveguides
}

\author{
Aitor V. Velasco*, Pavel Cheben, María L. Calvo*, Mirosław Florjańczyk, Przemek J. Bock, \\ André Delâge, Jens H. Schmid, Jean Lapointe, Siegfried Janz, Dan-Xia Xu and Martin Vachon \\ National Research Council Canada, Ottawa, Ontario, K1A 0R6, Canada \\ Tel: (001) 6139931624,Fax: (001)6139907656e-mail:Pavel.Cheben@nrc.ca \\ *Universidad Complutense de Madrid, 28040 Madrid, Spain \\ Tel: (0034) 913944629,Fax: (0034) 913944914,e-mail: avillafr@ucm.es
}

\begin{abstract}
We report a spatial heterodyne Fourier-transform spectrometer consisting of an array of Mach-Zehnder interferometers (MZI) implemented in silicon microphotonics. Optical path differences between the MZI arms increase linearly along the array, generating a wavelength-dependent interferogram which enables the retrieval of the source spectrum with a single measurement. Optical delays were implemented with Si-wire waveguides arranged in tightly coiled spirals to achieve a high resolution in a reduced footprint. Our spectral retrieval algorithm compensates phase and amplitude errors arising from fabrication imperfections by using a transformation matrix based on the calibration data. A wavelength resolution of $40 \mathrm{pm}$ within a free spectral range of $0.75 \mathrm{~nm}$ is demonstrated.
\end{abstract}

Keywords: spectroscopy, Fourier transform, silicon waveguides, spectral retrieval.

\section{INTRODUCTION}

Compact spectrometers with high-resolution and small footprint are required for a wide range of applications, including optical communications, biological and environmental sensing, and space instrumentation [1]. Additionally, a large optical throughput (étendue) is also required for the analysis of spatially extended and incoherent sources. Planar waveguide devices such as arrayed waveguide gratings (AWG) [2], echelle gratings $[3,4]$, lattice filters [5], ring resonators [6] and sidewall grating filters [7,8] provide high spectral resolution in a small device footprint, but are largely limited in terms of optical throughput. This limitation can be overcome by spatial heterodyne Fourier-transform (SHFT) spectrometers [9-12], which benefit from the intrinsically large étendue of the Michelson interferometer [13]. In particular, the SHFT concept can be implemented with an array of Mach-Zehnder interferometers (MZI) with linearly increasing optical path differences between MZI arms across the array [10]. This configuration provides a wavelength-dependent stationary interferogram from which the source spectrum can be retrieved by mathematical analysis.

Here we demonstrate a silicon waveguide SHFT spectrometer chip consisting of a MZI array with tightly coiled spiral structures [14]. A high spectral resolution of $40 \mathrm{pm}$ is demonstrated for a compact device with a footprint of $12 \mathrm{~mm}^{2}$. Furthermore, the disclosed spectral retrieval algorithm compensates phase and amplitude errors, circumventing the need of dedicated phase shifting circuits [15].

\section{OPERATION PRINCIPLE}

The SHFT microspectrometer is implemented in silicon-on-insulator (SOI) waveguides as an array of $N$ MachZehnder interferometers with linearly increasing optical path differences. Compact optical delays are achieved with Si-wire waveguides coiled in tight spirals, benefiting from the high mode confinement and small bend radius of the SOI platform. For a given input spectral distribution within the free spectral range (FSR) of an ideal device without phase errors, the MZI array generates a discretized stationary interferogram $Y\left(x_{i}\right)$ :

$$
Y\left(x_{i}\right)=\int_{0}^{F S R} B(\bar{\sigma}) \cos 2 \pi \bar{\sigma} x_{i} d \bar{\sigma}
$$

where $B$ is the incident spectral density, $x_{i}$ is the path delay of the $i$-th MZI, and $\bar{\sigma}=\sigma-\sigma_{L}$ is the shifted wavenumber, relative to the Littrow wavenumber $\sigma_{L}$ at which the maxima of the MZI transfer functions are aligned [10]. The input spectrum can be retrieved from the wavelength-dependent interferogram with a discrete cosine transform. Wavelength resolution $(\delta \lambda)$ and FSR of the spectrometer are determined by the maximum optical path difference $\left(\Delta L_{\max }\right)$ and the number of interferometers [14]:

$$
\begin{gathered}
\delta \lambda=\frac{\lambda_{0}^{2}}{\Delta L_{\max } n_{g}} \\
F S R=\delta \lambda \frac{N}{2}
\end{gathered}
$$

where $\lambda_{0}$ is the device operational central wavelength and $n_{g}$ is the waveguide group index. 


\section{DESIGN AND FABRICATION}

The spectrometer was designed with an array of 32 MZIs with spiral lengths linearly increasing from $365 \mu \mathrm{m}$ up to $\Delta L_{\max }=1.13 \mathrm{~cm}$ (Fig. 1). Si-wire waveguides with a width of $450-\mathrm{nm}$ were used to ensure monomode operation and bend losses minimization. Spirals were designed with a minimum bend radius of $5 \mu \mathrm{m}$ at their turning point, and a maximum diameter of $270 \mu \mathrm{m}$ for the spiral of the most unbalanced MZI. The device was nominally designed for TM polarization at the central wavelength of $1.55 \mu \mathrm{m}$. We used a layout with a single input waveguide at the facet followed by cascaded $y$-couplers. Nevertheless, independent input waveguides at chip facet for each MZI can be implemented for an increased étendue, maintaining identical operational principles and spectral retrieval techniques [10].

Devices were fabricated on $0.26-\mu \mathrm{m}$-thick silicon wafers with $2-\mu \mathrm{m}$ thick-buried oxide. Si-wire waveguide structures were defined by electron beam lithography using HSQ (hydrogen silsesquioxane) resist and transferred into the silicon layer by inductively coupled plasma reactive ion etching (ICP-RIE).

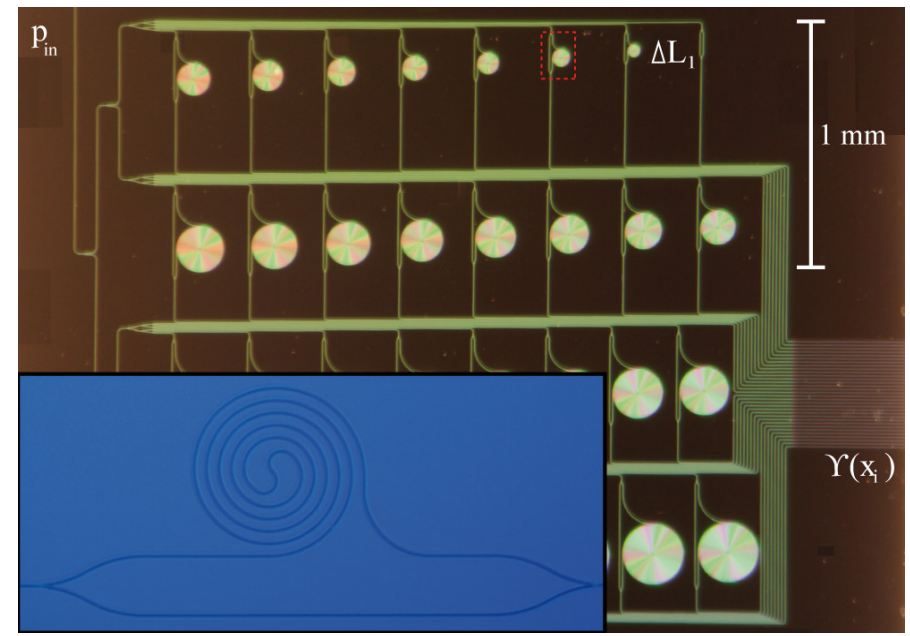

Figure 1. Optical micrograph of the fabricated spatial heterodyne Fourier-transform spectrometer chip with spiral Si-wire waveguides. Inset: magnified view of a Mach Zehnder interferometer.

\section{EXPERIMENTAL CHARACTERIZATION}

A high-resolution tunable semiconductor laser was used to characterize the fabricated device over the spectral range of $1550 \mathrm{~nm}-1552 \mathrm{~nm}$. Efficient subwavelength grating couplers [16] were included at the input and output facets of the chip for optimized fiber coupling and Fabry-Perot effect mitigation. The chip was thermally stabilized with a Peltier stage, and TM polarized input field was selected. The outputs of all the MZIs were measured simultaneously in a single shot with a high-sensitivity InGaAs photodetector array.

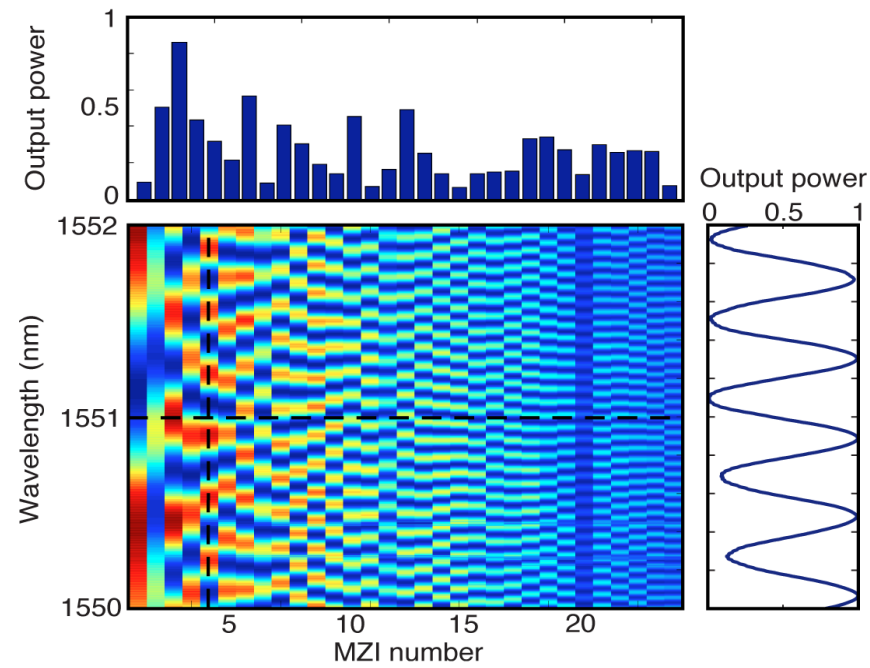

Figure 2. Experimental calibration measurement of the output power distribution of the 32 Mach-Zehnder interferometers for a $2 \mathrm{~nm}$ wavelength scan starting at $1.55 \mu \mathrm{m}$. The rows of the calibration matrix represent the normalized power of the output interferogram for each sampled wavelength (top), whereas the columns of the matrix comprise the spectral response of each Mach-Zehnder interferometer for coherent illumination (right). 
Figure 2 shows the experimental response of the $32 \mathrm{MZI}$ for the measured wavelength range, revealing two main deviations from the theoretical behavior given by Eq. 1. First, visibility $V$ of the MZI transmittance function varies along the array due to disparate path differences and insertion losses:

$$
V=\frac{I_{\max }-I_{\min }}{I_{\max }+I_{\min }}=\frac{2 \sqrt{I_{1} I_{2}}}{I_{1}+I_{2}}
$$

where $I_{\max }$ and $I_{\min }$ are the maximum and minimum output intensities of an interferometer, and $I_{1}$ and $I_{2}$ are the light intensities at the outputs of the two arms of the MZI, respectively. Second, the interferogram is distorted by phase errors arising from fabrication deviations in the length and effective index of the waveguides. As a consequence, MZI outputs are misaligned and the condition for the Littrow wavenumbers presence is not met by the array. Active compensation of this effect has been proposed in order to apply spectral retrieval algorithms based on discrete Fourier cosine transformation [15], however requiring dedicated phase shifting circuits and a complex operation.

\section{SPECTRAL RETRIEVAL}

Alternatively, our spectral retrieval algorithm includes calibration measurements of the MZI transmittance functions in a system of linear equations. A transformation matrix $T$ is defined such that $Y\left(x_{i}\right)=B \times T$ is verified for the calibration data. The source power spectrum $B$ is then retrieved by multiplying the spatial interferogram by a pseudo-inverse of the transformation matrix $T$, computed by Single Value Decomposition [17]. An apodization window is applied to reduce truncation ripple [18]. This procedure inherently compensates the influence of phase and amplitude errors by using the measured MZI transmittance functions instead of their ideal expressions. This presents a significant advantage against conventional cosine transform technique, which requires an active compensation of the phase errors [15], and against devices such as AWG with no direct physical access to the arrayed waveguide aperture [2].

Figure 3 demonstrates the spectral retrieval performed with our device and algorithm for a single monochromatic source (solid) and a doublet of two monochromatic lines separated $80 \mathrm{pm}$ (dashed). Experimental results show a resolution of $42 \mathrm{pm}$ (measured at full-width at half-maximum) and a FSR of $0.75 \mathrm{~nm}$.

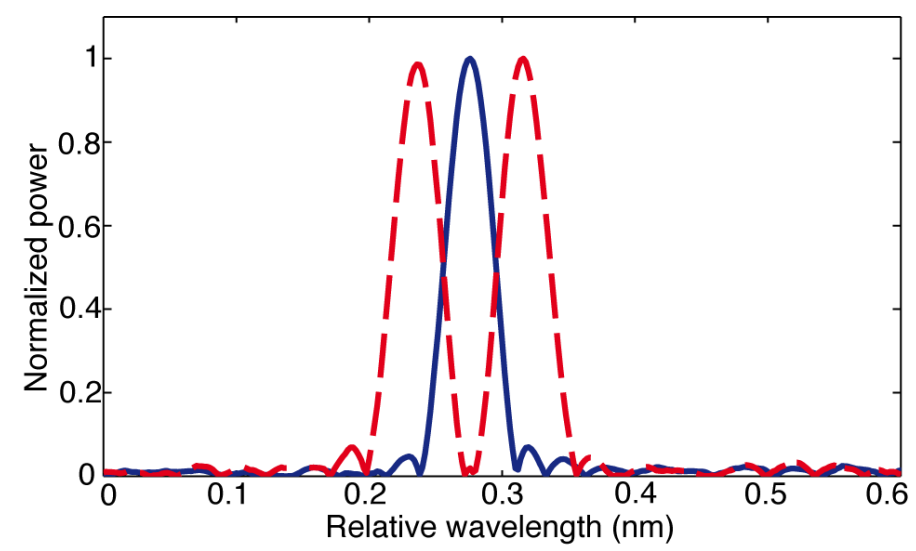

Figure 3. Spectra of a narrowband laser source (solid), and a doublet of two monochromatic lines separated $80 \mathrm{pm}$ (dashed), retrieved from experimental data with the spatial-heterodyne Fourier-transform spectrometer.

\section{CONCLUSIONS}

We have demonstrated a planar waveguide Fourier-transform spatial heterodyne spectrometer based on an interferometer array with Si-wire waveguides arranged in tightly coiled spirals. We have also presented a spectral retrieval technique with software compensation of phase and amplitude errors arising from fabrication deviations. A wavelength resolution of $40 \mathrm{pm}$ and a free spectral range of $0.75 \mathrm{~nm}$ have been demonstrated for a device with a maximum optical delay of $1.13 \mathrm{~cm}$ and a total footprint of $12 \mathrm{~mm}^{2}$. Potential applications of the microspectrometer include monitoring of optical communications, handheld instrumentation for biological and environmental sensing, and space-borne instrumentation for microsatellite platforms.

\section{ACKNOWLEDGEMENTS}

Financial support from the National Research Council and the Spanish Ministry of Economy is acknowledged under grants TEC2008-04105 and TEC2011-23629. 


\section{REFERENCES}

[1] P. Cheben, "Wavelength dispersive planar waveguide devices: Echelle gratings and arrayed waveguide gratings," in Optical Waveguides: From Theory to Applied Technologies, Eds. M. L. Calvo and V. Laksminarayanan, Chapter 5, CRC Press, London, 2007.

[2] P. Cheben, J. H. Schmid, A. Delâge, A. Densmore, S. Janz, B. Lamontagne, J. Lapointe, E. Post, P. Waldron, and D.-X. Xu, "A high-resolution silicon-on-insulator arrayed waveguide grating microspectrometer with submicrometer aperture waveguides," Opt. Express, vol. 15, pp. 2299-2306, 2007.

[3] S. Janz, A. Balakrishnan, S. Charbonneau, P. Cheben, M. Cloutier, A. Delâge, K. Dossou, L. Erickson, M. Gao, P.A. Krug, B. Lamontagne, M. Packirisamy, M. Pearson, and D.-X. Xu, "Planar waveguide echelle gratings in silica-on-silicon," IEEE Photon. Technol. Lett., vol. 16, pp. 503-505, 2004.

[4] J. Brouckaert, W. Bogaerts, P. Dumon, D. Thourhout, and R. Baets, "Planar concave grating demultiplexer fabricated on a nanophotonic silicon-on-insulator platform," J. Lightwave Technol., vol. 25, pp. 12691275, 2007.

[5] T. Mizuno, M. Oguma, T. Kitoh, Y. Inoue and H. Takahasi, "Lattice-form CWDM interleave filter using silica-based planar lightwave circuit", IEEE Photon. Technol. Lett., vol. 18, pp. 1570-1572, 2006.

[6] F. Xia, M. Rooks, L. Sekaric, and Y. Vlasov, "Ultra-compact high order ring resonator filters using submicron silicon photonic wires for on-chip optical interconnects," Opt. Express, vol. 15, pp. 1193411941, 2007.

[7] P. Bock, P. Cheben, J. Schmid, A. V. Velasco, A. Delâge, S. Janz, D.-X. Xu, J. Lapointe, T. J. Hall and M. L. Calvo, "Demonstration of a curved sidewall grating demultiplexer on silicon", Opt. Express, vol. 20, pp. 19882-19892, 2012.

[8] A. V. Velasco, P. J. Bock, P. Cheben, M. L. Calvo, J. H. Schmid, J. Lapointe, D.-X. Xu, S. Janz and A. Delâge, "Bandpass filter implemented with blazed waveguide sidewall gratings in silicon-on-insulator", Electron. Lett., vol. 48, pp. 715-717, 2012.

[9] P. Cheben, I. Powell, S. Janz, and D.-X. Xu, "Wavelength-dispersive device based on a Fourier-transform Michelson-type arrayed waveguide grating," Opt. Lett., vol. 30, pp. 1824-1826, 2005.

[10] M. Florjańczyk, P. Cheben, S. Janz, A. Scott, B. Solheim, and D. X. Xu, "Multiaperture planar waveguide spectrometer formed by arrayed Mach-Zehnder interferometers, “ Opt. Express, vol. 15, pp. 18176-18178, 2007.

[11] J. M. Harlander, F. L. Roesler, J. G. Cardon, C. R. Englert, and R. R. Conway, "A spatial heterodyne spectrometer for remote sensing of earth middle atmosphere” Appl. Opt., vol. 41, pp. 1343-1345, 2002.

[12] K. Okamoto, H. Aoyagi, and K. Takada, "Fabrication of Fourier-transform, integrated-optic spatial heterodyne spectrometer on silica-based planar waveguide," Opt. Lett., vol. 35, pp. 2103-2105, 2010.

[13] P. Jacquinot, "The luminosity of spectrometers with prisms, gratings, or Fabry-Perot etalons," J. Opt. Soc. Am., vol. 44, pp. 761-765, 1954

[14] A. V. Velasco, P. Cheben, P. J. Bock, A. Delâge, J. H. Schmid, J. Lapointe, S. Janz, M. L. Calvo, D.-X. Xu, M. Florjanczyk and M. Vachon, "High resolution Fourier-transform spectrometer chip with microphotonic silicon spiral waveguides," Opt. Lett., vol. 38, pp. 706-708 (2013).

[15] K. Takada, H. Aoyagi, and K. Okamoto, "Correction for phase-shift deviation in a complex Fouriertransform integrated-optic spatial heterodyne spectrometer with an active phase-shift scheme," Opt. Lett., vol. 36, pp. 1044-1046, 2011.

[16] P. Cheben, P. J. Bock, J. H. Schmid, J. Lapointe, S. Janz, D.-X. Xu, A. Densmore, A. Delâge, B. Lamontagne, and T. J. Hall, "Refractive index engineering with subwavelength gratings for efficient microphotonic couplers and planar waveguide multiplexers" Opt. Lett., vol. 35, pp. 2526-2528, 2010.

[17] G. H. Golub and C. Reinsch, "Singular value decomposition and least squares solutions", Numerische Mathematik, vol. 14, pp. 403-420, 1970.

[18] A. S. Filler, "Apodization and Interpolation in Fourier-transform spectroscopy", J. Opt. Soc. Am. vol. 54, pp. 762-767, 1964. 